\title{
MOLECULAR MODELING, ADMET PREDICTION, SYNTHESIS AND THE CYTOTOXIC ACTIVITY FROM THE NOVEL $N$-(4-tert-BUTYLPHENYLCARBAMOYL)BENZAMIDE AGAINST HELA
}

\author{
B.T. Purwanto ${ }^{1,} \bowtie$, Siswandono $^{1}$, D. Kesuma ${ }^{2}$, T. Widiandani ${ }^{1}$ and I. Siswanto ${ }^{3}$ \\ ${ }^{1}$ Department of Pharmaceutical Sciences, Faculty of Pharmacy, Universitas Airlangga, \\ Surabaya-60286, East Java-Indonesia \\ ${ }^{2}$ Department of Pharmaceutical Chemistry, Faculty of Pharmacy,Universitas Surabaya, \\ Surabaya-60293, East Java-Indonesia \\ ${ }^{3}$ Department of Chemistry, Faculty of Science and Technology, Universitas Airlangga, \\ Surabaya-60268, East Java-Indonesia \\ ${ }^{\circledR}$ Corresponding Author: bambangsutony@gmail.com
}

\begin{abstract}
Efforts to develop urea derivatives as anticancer agents thrive due to their proven anticancer activities. $N$-(4-tertbutylphenylcarbamoyl)benzamide was synthesized by Schotten Baumann reaction, rendering 59\%. To determine the purity, a thin-layer chromatography method in 3 different mobile phases was adopted, indicating a stain with distinguishing Rf. Structure identification indicating the compound was $N$-(4-tert-butylphenylcarbamoyl)benzamide. To predict cytotoxic activity, molecular docking with Autodocks Program was used by supplementing CHK1 enzyme (code PDB: 2YWP) against HeLa cells. The prediction score was confirmed by Molecular Docking Simulation (MDS). $N$-(4-tert-butylphenylcarbamoyl)benzamide (-4.41) has a smaller docking score compared to that of hydroxyurea (-2.69), suggesting higher cytotoxicity. MDS score highlighting this compound with ($13.1223 \pm 4.6818)$ total energy, smaller than the one in reference compound, $(-0.0446 \pm 0.3621)$. This new compound indicated properties with favorable anticancer activity in the ADMET prediction, due to the toxicity displayed against the organ. On the cytotoxic activity test against HeLa cells, the IC 50 of-(4-tertbutylphenylcarbamoyl)benzamide is $3.78 \mathrm{nM}$, smaller than that of hydroxyurea, $\mathrm{IC}_{50} 9.91 \mathrm{nM}$. The data suggest that the synthesized $\mathrm{N}$-(4-tert-butylphenylcarbamoyl) benzamidedisplays higher cytotoxic activity compared to hydroxyurea. This synthesized compound allows further study in drug development, serving as a new anticancer agent.
\end{abstract}

Keywords: Molecular Docking, Molecular Dynamic Simulation, ADMET Prediction, Synthesis, Cytotoxic Activity, $N$-(4-tert-butylphenylcarbamoyl)benzamide, HeLa Cells

RASĀYAN J. Chem., Vol. 14, No.2, 2021

\section{INTRODUCTION}

The imbalance between the production and scavenging of Reactive Oxygen Species (ROS) results in the disruption of normal cell function, leading to damage on protein, DNA, tissue, and biomolecule that induces the growth of new cells known as cancer cells. In the future, cancers will be more and more diagnosed, with lung and breast cancers rank the highest. According to National Breast Cancer Foundation in America, approximately 200,000 women were diagnosed with breast cancer. ${ }^{1}$

Cancer has become the second leading cause of mortality across the globe. As a result, coping with cancer is inevitably crucial in the field of medicine. The use of complementary and alternative drugs has become ubiquitous, encouraging rapid growth in drugs development, particularly in Asian countries. ${ }^{2}$

Modern drugs have successfully become an effective treatment for malignant tumors, and they are continuously developing. The failing therapy on cancers is primarily due to unspecific drug compounds, drug resistance, and the complications of the patients. Hydroxyurea is one of the cancer drugs working to inhibit the mitosis process of cancer cells and thus prevent them from proliferating. The urea protein 
RASĀYAN J. Chem.

Vol. 14 | No. 2 |1341-1350| April - June | 2021

structure as a pharmacophore allows other urea derivative compounds to display anticancer activity as well. ${ }^{3}$

Hydroxyurea has gained popularity as an anticancer drug. Yet, its ability to penetrate biological membranes is uncertain. The hydroxyl group bound to one of the nitrogen atoms turns the compound to be hydrophilic. Changing the structure of the urea compound is to improve the compound's penetration into a biological membrane. Adding a phenyl group to one of the nitrogen atoms will result in better penetration of this urea derivatives compound compared to hydroxyurea. The phenyl group, as in the $\mathrm{N}$ phenyl urea compound, will lead to a more lipophilic compound compared to hydroxyurea. Thus the compound can penetrate the biological membrane more easily. ${ }^{4}$

Some researchers have developed $N$-phenylurea and its derivatives as anticancer agents. Song et al. (2009) has synthesized 3-haloacylamino-phenylureas. Bromoacetil group bound to one of the hydrogen atoms exhibit potent anticancer activity against 8 human tumor cells with $\mathrm{IC}_{50} \mathrm{of} 0.38-4.07 \mathrm{uM}^{5}{ }^{5}$ Another study conducted by Szafranski et al. (2015) synthesized 4-substituted- $N$-(phenylcarbamoyl)-3-pyridine sulfonamide compound and its derivatives. One of the derivatives $N$-(4-chlorophenylcarbamoyl)-4(4-(3,4dichlorophenyl)piperazine-1-yl)pyridine-3-sulfonamide has potent antitumor activity against leukemia, colon cancer and melanoma with $\mathrm{IC}_{50}$ of 13.6-14.9 uM. ${ }^{6}$ In addition, another $N$-phenylurea derivative compound-1-(2-methyl-6-arylpyridine-3-yl)-3-phenylurea - has been synthesized with its 51 derivatives having potent anticancer activities against lung cancer A549 and colon cancer HCT-116 with $\mathrm{IC}_{50}$ of $3.22 \pm 0.2$ dan $2.71 \pm 0.16$ uM. $^{7}$

Predicting anticancer activity of $N$-phenylurea derivatives may adopt In-silico test molecular modeling with certain computer programs e.g. Autodock. This program allows investigating the reaction between the compound and the receptor or enzymes that play roles in the proliferation of cancer cells. One of these enzymes is Checkpoint kinase-1(CHK1) which contributes to the increasing damage of DNA on p-53 deficient cell. This DNA damage induces the phosphorylation process which terminates cell proliferation on phase S and G2. Therefore, the rise in Checkpoint kinase-1(CHK1) may expand DNA damage selectively on cancer cells, thus the existence of a selective inhibitor compound on CHKI is applicable for highly favorable cancer therapy. ${ }^{8}$ The compound prepared for a synthesis underwent an activity prediction test for its anticancer properties, through In-silico and molecular docking process against CHK-1 enzyme to obtain Molecular Docking Score (MDS) which would be compared with the standard compound, hydroxyurea. ${ }^{8}$ The ADMET prediction process was also required due to the novelty of $N$-(4-tertbutylphenylcarbamoyl)benzamidecompound to properly investigate the prediction score of absorption, distribution, metabolism, excretion and also the toxicity. This enables further development of the compound as a novel anti-cancer agent. ${ }^{9}$

$N$-(4-tert-butylphenylcarbamoyl)benzamide, the synthesized $N$-phenylurea derivative compound, was derived from nucleophilic acylation between $N$-phenylurea and 4-tert-butylbenzoyl chloride using Schotten Baumann method..$^{10}$ The physical and chemical properties of the synthesized compound changed in the process. The lipophilic, electronic and steric properties turned higher compared to the original compound, thus enhancing the anticancer activity of the synthesized compound. ${ }^{4}$

By applying the MTT assay method and using HeLa cancer cells - standard cells most commonly used in anticancer activity test - anticancer activity test was performed. ${ }^{11}$

\section{EXPERIMENITAL}

\section{Material}

Materials for physicochemical synthesis and analysis: $N$-phenylurea, 4-t-butylbenzoyl chloride, pyridine, variants of organic solvent (acetone, ethyl acetate, $n$-hexane, chloroform, ethanol, and methanol with pro analysis quality), Kieselgel $60 \mathrm{~F}_{254}$, DMSO-d 6 .

Materials for activity test: Synthesized compound, HeLa culture cell, Culture Media DMEM. DMSO, Phosphate-buffered Saline (PBS), MTT [3-(4,5-dimethylthiazol-2-il)-2,5-diphenyltetrazolium bromide], SDS $10 \%$ in $0,1 \mathrm{~N} \mathrm{HCl}$.Standard compound: hydroxyurea (Sigma Aldrich).

The Tools used for Synthesis and Analysis of Compound Structure

Glass tube, UV-vis Shimadzu HP 8452 ASpectrophotometer, Jasco FT-IR 5300 Spectrophotometer, NMR 
RASĀYAN J. Chem.

Vol. 14 | No. 2 |1341-1350| April - June | 2021

Hitachi R-1900Spectrometer, Electrothermal Mel-Temp, Corning Hot Plate P 351, Shimadzu LM-20 analytical balance.

\section{Tools for Cytotoxic Test}

Micropippet 200,1000 $\mu \mathrm{L}$ and tip, culture tube, microplate, Conical tube, ELISA-Reader.

\section{Devices and Software used for Molecular Modelling}

Lenovo computer, operating system Windows 10, 64 bit, intel core i-5-7200 U, CPU@250 GHz, 8.00 GB RAM. Mgl Tools (Version 1.5.6). AutoDockTools (Version 4.2.6) with Autogrid.

\section{Devices used for ADMET Prediction}

Lenovo computer, operating system Windows 10, 64 bit, intel core i-5-7200 U, CPU@250 GHz, 8.00 GB RAM. Chem.Bio.Draw Ultra Version 12 (Cambridge Soft); Chem.Bio3D Ultra Version 12 (Cambridge Soft); Online SMILES Translator, and pkCSM online tool.

\section{Docking Method Validation}

When doing docking protocol on $\mathrm{N}$-(4-tert-butylphenylcarbamoyl)benzamidecompound using Autodock 4.2.6 dan Autogrid program, it is required to perform method validation by redocking its native ligand to A41_1 protein, whose native ligand has been removed. The parameter for this method validation is the value of Root Mean Square Deviation (RMSD), with a tolerable value of $\leq 2.0 \mathrm{~A}^{\mathrm{o}} .^{12}$

\section{In-silico Prediction on Cytotoxic Activity}

Autodocks software (Version 4.2.6) was used to predict the cytotoxic activity of this compound. Molecular docking simulation is performed between the test compound and checkpoint kinase 1 enzyme (CHK1), ID PDB: 2YWP which contains a ligand of 1-(5-chloro-2,4-dimethoxyphenyl)-3-(5cyanopyrazin-2-yl)urea (A42_1), downloaded from Protein Data Bank (PDB) server.

To prepare protein and ligand molecule, Mgl Tools (Version 1.5.6) was used. And to obtain a perfect/great assignment grid parameter of each ligand, the grid box was generated by trials and errors.

The grid box dimension of X: 32, Y: 20, Z: 16, while the grid box center of X: -3.864, Y: 9.389, Z: 18.202 were used for docking calculation. Subsequently, the docking process was conducted by running the Lamarckian Genetic Algorithm. Here, the default parameters and one hundred independent docking runs were carried out for every chemical structure. ${ }^{13-17}$

\section{Molecular Dynamics Simulation}

The ligand-receptor binding energy that has been calculated using molecular docking procedure should allow validations on molecular dynamics simulation. ${ }^{18}$ This simulation procedure adopts the Amber Molecular Dynamics program package for 10ns simulation. ${ }^{19}$ The trajectory files from this simulation were analyzed to calculate free energy binding between receptors and each of the ligands. To calculate the simulation, Python interpreter module and MMPBSA.py in Amber Molecular Dynamics Package Program were employed. Before calculating, the complex bindings between the receptor of checkpoint kinase1 and the native ligand model (PDB ID: 2YWP) were downloaded from the PDB server. This process worked with the UCSF Chimera program. ${ }^{20}$ Partial atomic charges were added to each standardized residual of the receptor using Molecular Mechanics (MM) software with AMBER ff14SB force field. While for non-standardized residuals of the ligand, the semi-empirical AM1-BCC method was applied. ${ }^{21}$ Other ligands in this experiment - 4TBPCB, and HU were converted from their 2D to 3D ligands using the semi-empirical PM6 method.

\section{ADMET Prediction}

The prediction on pharmacokinetic properties (ADMET: Absorption, Distribution, Metabolism, Excretion and Toxicity) of the active compound was determined using pkCSM online tool software. The 2D structure of the active compound was illustrated in Chem. Bio. Draw. Ultra-Version 12.0 programe. To obtain the 3D image of the structure, the illustration was copied to Chem. Bio. 3D Ultra Version 12.0 
RASĀYAN J. Chem.

Vol. 14 | No. 2 |1341-1350| April - June | 2021

software and saved on ${ }^{*}$.sdf files and subsequently interpreting the active compound data on SMILE format in Online SMILES translator (http://cactus.nci.nih.gov/translate/). In its SMILES format, the active compound underwent ADMET prediction by utilizing pkCSM online tool (htpp://biosig.unimelb.edu.au/pkCSM/prediction). ${ }^{22,23}$

\section{Synthesis of-(4-tertier-butylphenylcarbamoyl) benzamide Compound}

$0,03 \mathrm{~mol}$ of $\mathrm{N}$-phenylurea compound was mixed with $40 \mathrm{ml}$ of tetrahydrofuran and pyridine $4 \mathrm{ml}$ on a 200 in a round-bottom flask. 4-t-butylbenzoyl chloride $0,01 \mathrm{~mol}$ was added at $5^{\circ} \mathrm{C}$ temperature into $20 \mathrm{ml}$ of tetrahydrofuran and placed on a magnetic stirrer hotplate. Once 4-t-butylbenzoyl chloride solution was completely heated up, the mixture was refluxed and stirred for 8 hours.

Upon terminating the reaction, tetrahydrofuran was then evaporated on rotavapor. Saturated natrium bicarbonate liquid was supplemented on the reaction outcome and stirred to eliminate any bubbles. Using Buchner filter funnel, the solid substance was rinsed with $50 \mathrm{ml}$ water and repeated twice, followed by a wash with $10 \mathrm{ml}$ ethanol two times. The recrystallization process was performed by diluting the solid substance with hot ethanol adequately while stirred in a hot plate. The solution was filtered while hot and stored at room temperature overnight. The crystallized substance was filtered with a Buchner funnel, washed in $10 \mathrm{ml}$ ethanol twice. Recrystallization can also be conducted using another appropriate solution depending on the synthesized compound, like acetone-water. The crystal formed was transferred to a petri dish, dried in a $50^{\circ} \mathrm{C}$ oven to obtain a constant mass. ${ }^{24-27}$

The structure identification of active N-(4-tert-butylphenylcarbamoyl)benzamide utilized the following instruments: UV-Vis dan IR spectrophotometer, ${ }^{1} \mathrm{H}-\mathrm{NMR}$, dan Mass spectrometer. ${ }^{28}$

The reaction between $\mathrm{N}$-phenylurea with 4-t-butylbenzoyl chloride compound can be seen in Fig.-2 as follows:<smiles>CC(C)(C)c1ccc(C(=O)NC(=O)Nc2ccccc2)cc1</smiles>

Fig.-2: The Synthesis Reaction of $N$-(4-tert-butylphenylcarbamoyl)benzamide

\section{Cytotoxic Activity Test}

Anticancer activity or cytotoxic test in vitro with cancer cells were proceeded with MTT assay approach. A mother compound was prepared from the test compound, $N$-(4-tert-butylphenylcarbamoyl)benzamide, as much as $5000 \mu \mathrm{g} / \mathrm{mL}$ in DMSO solution. From each mother compound, a series of standard compounds with $250,500,750,1000,1500,2000 \mu \mathrm{g} / \mathrm{mL}$ concentrations were prepared by dilution. As a positive control, standard anticancer drug solution, hydroxyurea was prepared along with solvent blanks as a negative control.

The culture of cancer cells (HELA cells) was prepared, and so with normal cells in cell suspension with $10^{5}-2.10^{6}$. density. Each $100 \mu \mathrm{L}$ of the cells were seeded in Microplate wells, except those for control media. $0,2 \mathrm{~mL}$ of standard solution, positive and negative control, each was added in a microplate well. Each standard solution was replicated 3 times. The microplate was incubated for 24 hours in $5 \% \mathrm{CO}_{2}$ incubator at $37^{\circ} \mathrm{C}$ with $\mathrm{pH}$ 7.4-7.7.

MTT reagent was prepared for $0,5 \mathrm{mg} / \mathrm{ml}$ treatment by diluting $1 \mathrm{~mL}$ of MTT stock (there is $50 \mathrm{mg}$ MTT in $10 \mathrm{~mL}$ PBS) with adequate media. After incubation, cell media was removed, and cells were washed in PBS then added with $100 \mu \mathrm{L}$ of MTT reagent on each well, including control media (no cells included). The plate was incubated for 24 hours. Once formazan was formed, it was added with $100 \mu \mathrm{L}$ SDS $10 \%$ in $0,1 \mathrm{~N} \mathrm{HCl}$. The plate was covered with aluminium paper and incubated in a dark room at room temperature for 24 hours. The absorbance of each well was examined in an ELISA reader at $595 \mathrm{~nm}$ 
RASĀYAN J. Chem.

Vol. 14 | No. 2 |1341-1350| April - June | 2021

wavelength. The more cells proliferate, the bigger is the absorbance. $\mathrm{The} \mathrm{IC}_{50}$ of the test compound was determined with probit regression analysis. ${ }^{29}$

\section{Docking Validation Score}

\section{RESULTS AND DISCUSSION}

After performing the validation method by the redocking native ligand on A42_1 protein, whose native ligand has been removed, Root Mean Square Deviation (RMSD) value was obtained: $1.02 \mathrm{~A}^{\mathrm{o}}$. The tolerable RMSD value is $\leq 2.0 \mathrm{~A}^{\circ}$.

In this study, as indicated in Table-1, the average value of RMSD of a native ligand with A42_1 is 1.02 . This means Autodock 4.2 and Autogrid method have met the requirements for validation.

\section{In-silico Molecular Modeling}

The result of molecular docking of standard ligand compound 1-(5-Chloro-2,4- dimethoxy-phenyl) -3-(5cyanopyrazin-2-yl)urea, reference compound Hydroxyurea and test compound $N$-(4-tertbutylphenylcarbamoyl)benzamideusing Autodock 4.2.6 program can be seen in Table-1.

Table-1: Molecular Docking Score on Standard Ligand Compound, Hydroxyurea and N-(4-tertbutylphenylcarbamoyl)benzamide using Autodock 4.2.6 Program

\begin{tabular}{|c|c|c|}
\hline \multirow{2}{*}{$\begin{array}{l}\text { Compound } \\
\text { 1-(5-Chloro-2,4-dimethoxyphenyl)- } \\
\text { 3-(5-cyanopyrazin-2-yl)urea } \\
\text { RMSD : } 1.02 \mathrm{~A}^{\circ}\end{array}$} & MDS & Interactions of Ligand-amino acid \\
\hline & -6.85 & 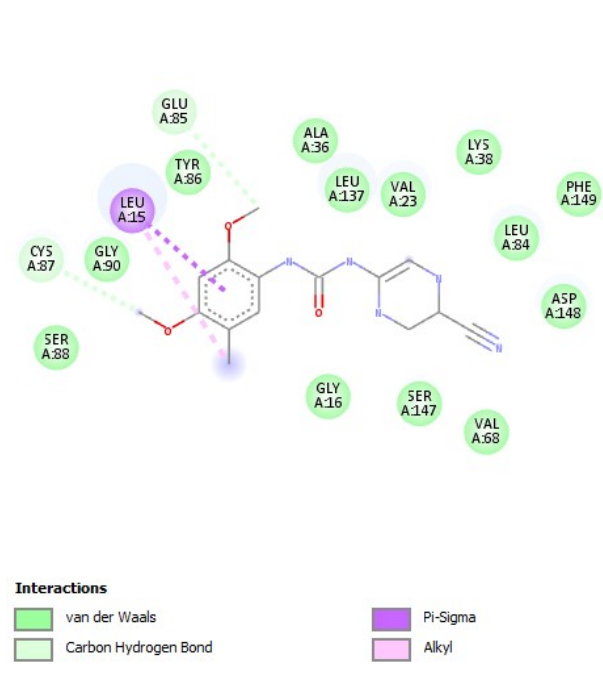 \\
\hline Hydroxyurea & -2.69 & Interctions \\
\hline
\end{tabular}


RASĀYAN J. Chem.

Vol. 14 | No. 2 |1341-1350| April - June | 2021

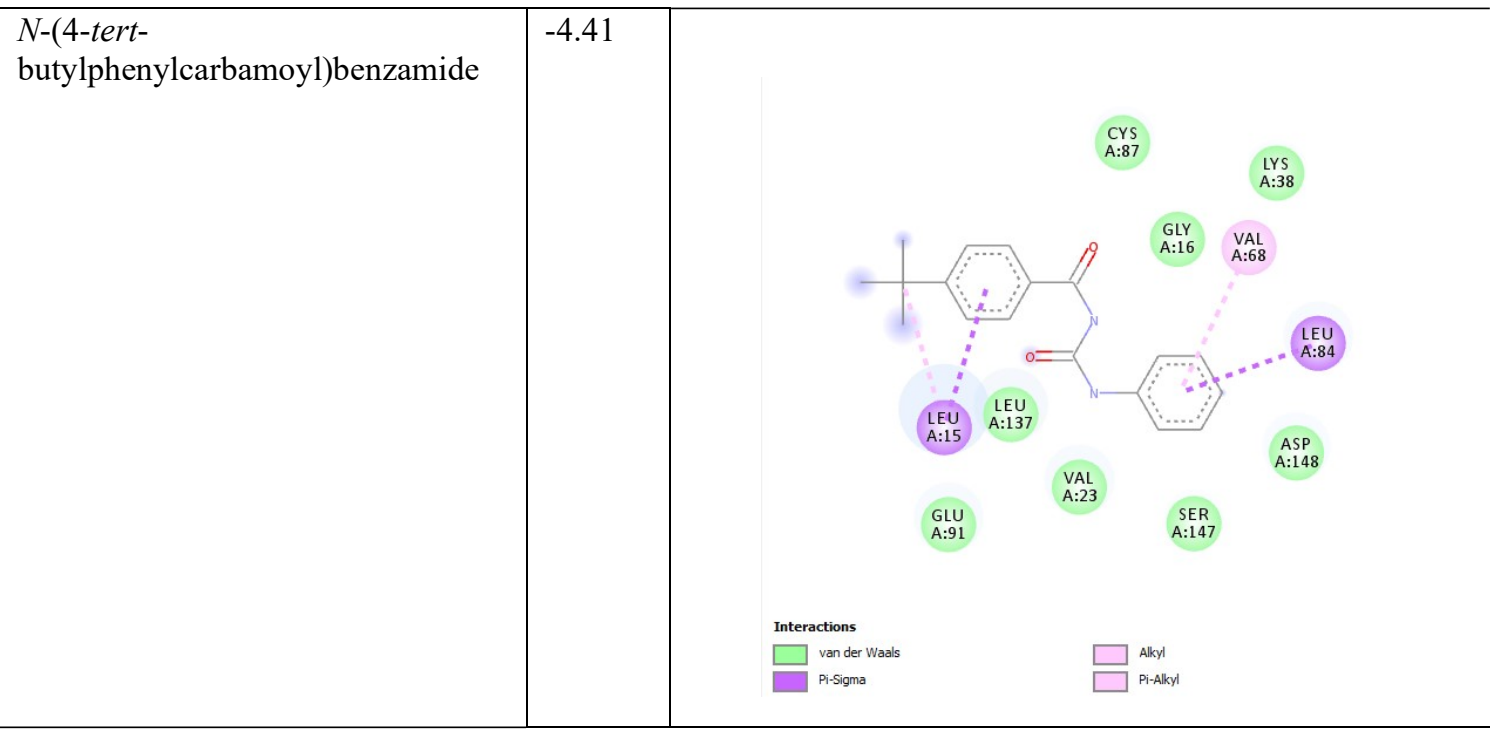

Interactions between ligand molecules and receptors were as displayed in Table-1. N-(4-tertbutylphenylcarbamoyl)benzamide compound demonstrate docking score of -4.41 , smaller compared to that of hydroxyurea, -2.69. This indicates that the binding between $N$-(4-tertbutylphenylcarbamoyl)benzamidewith its receptor is substantially more stable due to the smaller energy required. In addition, there are a lot more bindings between $N$-(4-t-butylphenylcarbamoyl)benzamide compound and amino acid proteins ( CysA:87; LysA:38; GlyA:16; ValA:58; LeuA:84; AspA:148; SerA:147; Val A:23; LeuA:137; Leu A:15; GluA:91) compared to those with hydroxyurea (ValA:23; LeuA:15; Tyr A:86; GluA:85; Ala A:36; CysA:87; LeuA;137). Thus, the binding between $N$-(4-t-butylphenylcarbamoyl)benzamide with the receptors will be stronger. Therefore, it is predicted that $N$-(4-tertbutylphenylcarbamoyl)benzamidehas higher cytotoxic activity than that of hydroxyurea. However, when compared to the native ligand, A42_1, N-(4-tert-butylphenylcarbamoyl)benzamidehas a greater MDS value. Hence, it is predicted that this compound has less cytotoxic activity.

\section{Molecular Dynamics Simulation}

Binding free energy calculated from this simulation for hydroxyurea (HU) and $N$-(4-tertbutylphenylcarbamoyl)benzamide (4TBPCB) was listed in Table-2.

Table-2: Binding Free Energy between Receptor and Ligands after 10 ns Simulation

\begin{tabular}{c|c|c}
\hline \multirow{2}{*}{ Energy Component } & \multicolumn{2}{|c}{ Ligands } \\
\cline { 2 - 3 } & 4TBPCB & HU \\
\hline VDWAALS & $-14.8863 \pm 5.0141$ & $-0.0931 \pm 0.4101$ \\
\hline EGB & $3.7761 \pm 1.1997$ & $0.0675 \pm 0.1999$ \\
\hline ESURF & $-2.0121 \pm 0.6470$ & $-0.0190 \pm 0.1092$ \\
\hline DELTA G gas & $-14.8863 \pm 5.0141$ & $-0.0931 \pm 0.4101$ \\
\hline DELTA G solv & $1.7641 \pm 0.7831$ & $0.0485 \pm 0.1223$ \\
\hline DELTA Total & $-13.1223 \pm 4.6818$ & $-0.0446 \pm 0.3621$ \\
\hline
\end{tabular}

To substantiate the prediction of cytotoxic activity of $N$-(4-tertbutylphenylcarbamoyl)benzamidecompound, Molecular Dynamic Simulation (MDS) was performed as displayed in table 2. $N$-(4-tert-butylphenylcarbamoyl)benzamide has a component total energy of 13.1223, far smaller compared to that of hydroxyurea, -0.0446 . This confirms the prediction that the cytotoxic activity of $N$-(4-tert-butylphenylcarbamoyl) benzamide is higher than that of hydroxyurea.

\section{ADMET Prediction}

ADMET prediction score of reference compound hydroxyurea (HU) and test compound $N$-(4-tertbutylphenylcarbamoyl)benzamide (4TBPCB) can be seen in Table-3. 
RASĀYAN J. Chem.

Vol. 14 | No. 2 |1341-1350| April - June | 2021

Table-3: ADMET Prediction of Reference Compound hydroxi urea (HU) and Test Compound N-(4-tertbutylphenylcarbamoyl)benzamide(4TBPCB)

\begin{tabular}{c|c|c|c}
\hline ADMET & Test & HU & 4TBPCB \\
\hline \multirow{2}{*}{ Absorption } & Intestinal Absorption (human) in $\%$ & 73.127 & 90.931 \\
& Skin Permeability (log Kp, cm $/ \mathrm{h})$ & -4.319 & -3.08 \\
\hline \multirow{2}{*}{ Distribution } & VDss $(\log \mathrm{L} / \mathrm{kg})$ & -0.495 & -0.238 \\
& BBB Permeability $(\operatorname{logBB})$ & -0.545 & 0.365 \\
\hline \multirow{2}{*}{ Metabolism } & CYP2D6 Substrate & No & No \\
& CYP2D6 Inhibitor & No & No \\
\hline \multirow{2}{*}{ Excretion } & Renal OCT2 Substrate & 0.659 & No \\
& Total Clearance (log ml $/ \mathrm{min} / \mathrm{kg})$ & Yes & 0.234 \\
\hline \multirow{2}{*}{ Toxicity } & Ames Toxicity & 2.116 & 1.611 \\
& LD & No & No \\
& Hepatotoxic & & \\
\hline
\end{tabular}

As many as 2.245 drug compounds were analyzed by Lipinski et al., and listed in World Drug Index and known as Lipinski rule of 5 since the resulted analysis values are multiples of Five. When a drug compound has a molecular weight $>500$, it will be difficult to be absorbed with low permeability. The Partition Coefficient Log value on octanol/water $(\log \mathrm{P})$ is bigger than +5 , hydrogen binding donor value (HBond=HBD) was expressed by the number of bindings of $\mathrm{OH}$ and $\mathrm{NH}$ groups, more than 5 , and Hydrogen receptor (HBO) expressed by the number of $\mathrm{O}$ and $\mathrm{N}$ atoms, more than 10.30 .

$N$-(4-tert-butylphenylcarbamoyl)benzamidecompound has a molecular weight of 296.36, smaller than 500 , with Partition Coefficient $\log$ value on octanol/water $(\log \mathrm{P})=4.16$, smaller than +5 . The number of $\mathrm{OH}$ and $\mathrm{NH}$ groups $=2$, less than 5, while the number of $\mathrm{O}$ and $\mathrm{N}$ atoms $=4$, less than 10 . Hence, this compound is predicted to have great absorption and high permeability.

After administering oral medication, drugs are normally best absorbed in the intestine. Here, the number of absorbed compounds can be predicted. When less than $30 \%$, it means the absorption is poor. ${ }^{30}$ Ideally, drug compounds have $90 \%$ of oral bioavailability with no individual variability. ${ }^{31}$ In Table-3, $N$-(4-tertbutylphenylcarbamoyl)benzamideseems to have human intestinal absorption of $90.931 \%$, meaning that the compound is predicted to have good bioavailability.

For transdermal drug development, skin permeability is compelling to study. Skin permeability coefficient is stated with $\mathrm{Kp}(\mathrm{cm} / \mathrm{h}) \log$ value that allows skin permeability prediction on drug compound. One compound is predicted to have skin permeability when the $\mathrm{Kp}(\mathrm{cm} / \mathrm{h}) \log$ value $\mathrm{Kp}>-2.5$. In Table3, $N$-(4-t-butyl-phenylcarbamoyl) benzamide compound has log value of $\mathrm{Kp}-3.08(\mathrm{~cm} / \mathrm{h})$, which means that this compound has low permiability. ${ }^{32}$

The volume of distribution (VDss) is the theoretical volume of drugs total dosage required for distribution, thus enables equal concentration in blood. When a compound is high in VDss value, it can be said that the compound is distributed more in the tissue than in blood plasma. When the log value is VDss $<-0.15$, it suggests a low distribution. When the log value is VDss $>0.45$ then it has high distribution. ${ }^{32}$

In table 3, $N$-(4-tert-butylphenylcarbamoyl)benzamidecompound is shown to have log value VDss -0.238 , meaning that this compound has a low distribution volume.

According to Pires et al (2015), a compound is considered capable of penetrating the blood-brain barrier well when it has $\log \mathrm{BB}>0,3$. On the other hand, with $\log \mathrm{BB}<-1$ a compound is unable to distribute well. In Table-3, $N$-(4-tert-butyl-phenylcarbamoyl)benzamide compound indicates a value of $\log \mathrm{BB}$ 0.365 . Thus, it can be inferred that this compound is incapable of penetrating the blood-brain barrier. ${ }^{32}$

Cytochrome P450 is an important enzyme in the detoxification process and is the primary enzyme located in the liver. This enzyme plays a significant role in the oxidation process and may facilitate excretions of foreign organic compounds including drugs. It is crucial to note that drug compounds may inhibit the cytochrome P450 enzyme from functioning. In this study, it is represented by Cytochrome P3D6 isofarm (CYP2D6). $N$-(4-tert-butylphenylcarbamoyl)benzamidecompound in Table-3 indicates that it does not inhibit the function of the cytochrome P450 enzyme.

The excretion process of drug compounds is possible to predict by measuring the total clearance (Cltot) and Renal Organic Cation Transporter 2 (OCT2) substrate. C1 total is a combination of hepatic clearance 
RASĀYAN J. Chem.

Vol. 14 | No. 2 |1341-1350| April - June | 2021

(metabolism in liver and bile) and renal excretion. In table 3, $N$-(4-tert-butylphenylcarbamoyl)benzamide compound has a value of 0.234 which indicates the excretion speed of the compound.

OCT2 is a transporter in the kidney that has an important role in the disposition and clearance of drug compounds and endogen compounds in the human body. $N$-(4-tertbutylphenylcarbamoyl)benzamidecompound in Table-3 demonstrates that this compound does not affect OCT 2 , enabling the compound to excreted so easily. ${ }^{32}$

Ames cytotoxic test is an assay to demonstrate the toxicity of a compound and is commonly adopted to access the mutagenic nature of a compound using bacteria. When tested positive, it suggests that the compound is mutagenic dan possibly act as a carcinogen. $N$-(4-tert-butylphenylcarbamoyl)benzamidein Table-3 displays that this compound is nontoxic both in Ames test and hepatotoxicity. Hence, this compound is considered safe. In a compound with $\mathrm{LD}_{50}$ test on mice, the value is $1.611 \mathrm{mg} / \mathrm{kgBW}$, suggesting that the compound has a low toxicity value. To kill a guinea pig $50 \%$, it takes $457 \mathrm{mg} / \mathrm{kgBW}$ dosage. ${ }^{32}$

\section{Chemical Structure Identification}

$N$-(4-tertier-butylphenylcarbamoyl)benzamide, yield: $59 \%$; MP: $171^{\circ} \mathrm{C}$; UV (methanol, $\lambda$ maks, nm): 236 ; FT-IR (KBr pellet, $\mathrm{cm}^{-1}$ ): 3467 (NH sec), 2965 (C-H alkane), 1685 and 1610 (2 C=O ureide), 1568, 1423 $\left(\mathrm{C}=\mathrm{C}\right.$ arom); ${ }^{1} \mathrm{H}-\mathrm{NMR}$ (DMSO- $\left.\mathrm{d}_{6}, \sigma, \mathrm{ppm}\right): 7.00-8.20\left(\mathrm{~m}, 9 \mathrm{H}, \mathrm{C}_{6} \mathrm{H}_{5}\right.$ and $\left.\mathrm{C}_{6} \mathrm{H}_{4}\right) ; 10.20(\mathrm{~s}, 1 \mathrm{H}, \mathrm{NH}) ; 10.80$ (s, $1 \mathrm{H}, \mathrm{NH}) ; 1.0-1.6\left(\mathrm{~m}, 9 \mathrm{H}, \mathrm{C}\left(\mathrm{CH}_{3}\right)_{3}\right.$; MS (EI; m/e): $246(\mathrm{M})^{+}$.

According to the interpretation data of compound structure in item 4 , where there are 2 carbonyl ureida groups on wave number 1685 and $1610 \mathrm{~cm}^{-1}$ on Infra-Red interpretation. This is then confirmed with 9 hydrogen atoms on 7.00-8.20 ppm chemical shifton ${ }^{1} \mathrm{H}-\mathrm{NMR}$ spectrum. In addition, a molecular weight of the compound interpreted on Mass Spectral Method (246) shows that $N$-(4-tertbutylphenylcarbamoyl)benzamidecompound has been successfully synthesized and has structures as depicted in Fig. -5 below.

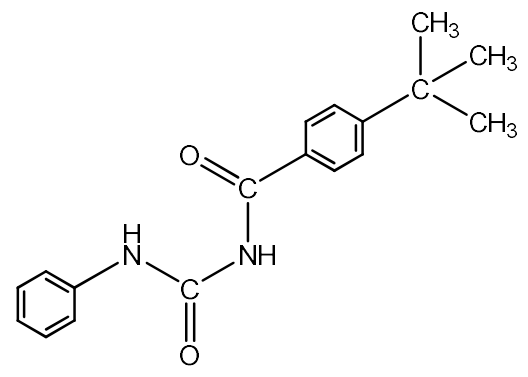

Fig.-5: Structure of $N$-(4-tert-butylphenylcarbamoyl)benzamide

\section{Cytotoxicity Test with HeLa Cells}

Data of cytotoxicity test with HeLa cells from reference compound Hydroxyurea and test compound $\mathrm{N}$ (4-tert-butylphenylcarbamoyl)benzamide are shown in Figs.-3 and 4 and Table-4.

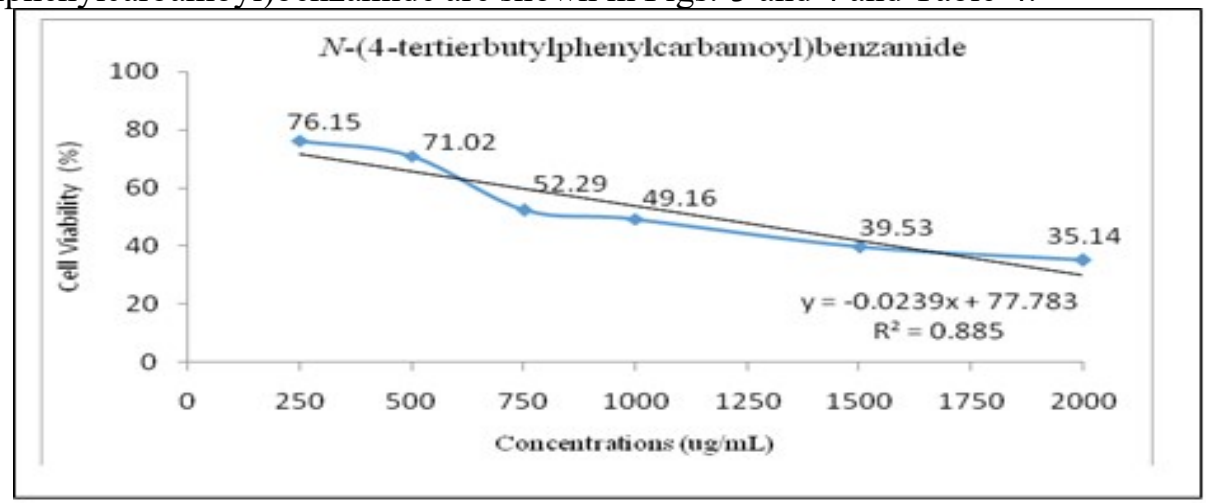

Fig.-3: Cytotoxic Activity Curve of N-(4-tert-butylphenylcarbamoyl)benzamide Compound 
RASĀYAN J. Chem.

Vol. 14 | No. 2 |1341-1350| April - June | 2021

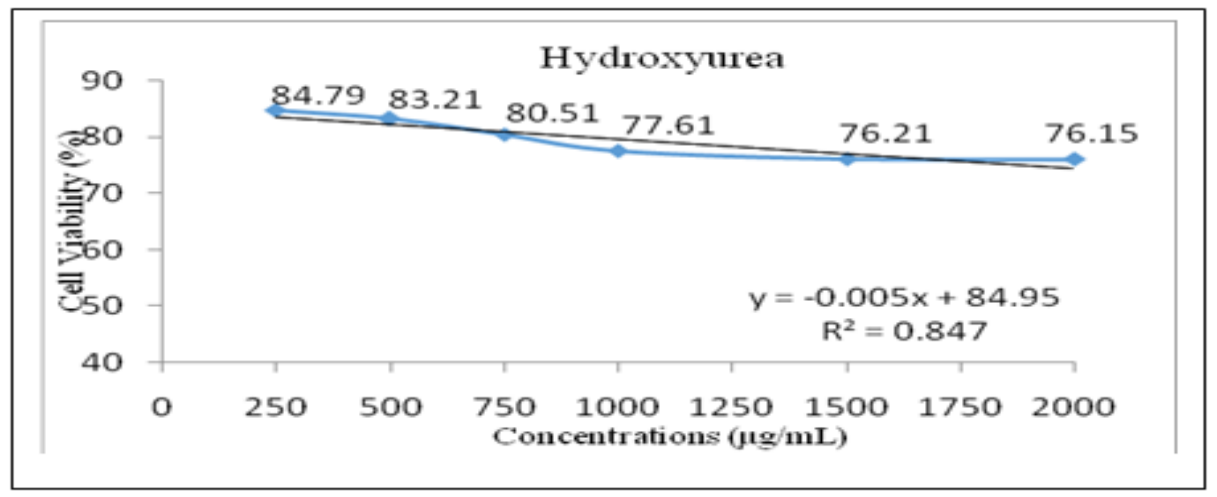

Fig.-4: Cytotoxic Activity Curve of Hydroxyurea

Table-4: Data of Cytotoxicity Test with HeLa Cells from Reference Compound Hydroxyurea and Test Compound $\mathrm{N}$-(4-tert-butylphenylcarbamoyl)benzamide

\begin{tabular}{c|c|c}
\hline \multirow{2}{*}{$\begin{array}{c}\text { Dosages } \\
(\mu \mathrm{g} / \mathrm{ml})\end{array}$} & \multicolumn{2}{|c}{ \%Viability Cells } \\
\cline { 2 - 3 } & Hydroxy Urea & 4TBPCB \\
\hline 250 & 84.79 & 76.15 \\
\hline 500 & 83.21 & 71.02 \\
\hline 750 & 80.51 & 52.29 \\
\hline 1000 & 77.61 & 49.16 \\
\hline 1500 & 76.21 & 39.53 \\
\hline 2000 & 76.15 & 35.14 \\
\hline
\end{tabular}

According to the data shown in table 3 for $N$-(4-tert-butylphenylcarbamoyl)benzamide compound and hydroxyurea, curve image on figure 2 and curve image on figure 3, it can be seen that $N$-(4-tertbutylphenylcarbamoyl)benzamide compound has higher cytotoxic activity compared to hydroxyurea. On a conducted regression analysis, the $\mathrm{IC}_{50}$ of $\mathrm{N}$-(4-tert-butylphenylcarbamoyl)benzamide compound obtained is $9.1 \mathrm{mM}$. It can be inferred that $N$-(4-tert-butylphenylcarbamoyl)benzamide compound has far greater cytotoxicity compared to hydroxyurea.

\section{CONCLUSION}

$N$-(4-tert-butylphenylcarbamoyl)benzamide compound has been synthesized and has a great cytotoxic activity, allowing further study in drug development and serves as a new anticancer agent.

\section{REFERENCES}

1. M. Sidharta, G. R. Kumar, B. Prasanna and A. Thirupathiah, Rasayan Journal Chemistry, 11(2), 661(2018), DOI:10.37188/ RJC.2018.1122062

2. V.S. Talismanov, S.V. Popkov, S.S. Zykova, O.G. Karmanova, S.A. Bondarenko, Journal of Pharmaceutical Scences and Research, 10(1),152(2018).

3. P. Gummala, A. Annapura, P. Madhukiran, International Journal of Green Chemistry, 11(4), 230(2017).

4. Siswandono, Pengembangan Obat Baru, Surabaya: Airlangga University Press (2016)

5. D.Q. Song, N.N. Du, Y.M. Wang, W.Y. He, E.Z. Jiang, S.X. Cheng, Y.X. Wang, Y.H. Li, Y.P. Wang, X. Li, J.D. Jiang, Bioorganic Medicinal Chemistry, 17(11), 3873(2009), DOI:10.1016/j.bmc.2009.04.022

6. S. Krysztof and Joroslawinski, Molecules, 20, 12029(2015), DOI:10.3390/molecules.200712029

7. W.M. Eldehna, H. Ghada, A. Rashad. T. Sora, A.W. Tarfah., A. Ahmed, A.K. Hamad, A.A. Abdulrahman., A.H.A. Abdel, Journal of Enzyme Inhibition and Medicinal Chemistry, 34(1), 322(2019), DOI: 10.1080/14756366.2018.1547286

8. A. Hincliffe, Molecular Modelling for the beginner, $2^{\text {nd }}$ Ed, Chichester: John Wiley and Sons., Ltd. (2008) 
RASĀYAN J. Chem.

Vol. 14 | No. 2 |1341-1350| April - June | 2021

9. S. Hardjono, T. Widiandani, B.T. Purwanto, L.A. Nasyanka, Research Journal Pharmaceutical and Technology, 12(5), 2160(2019), DOI:10.5958/0974-360X.2019.00359.7

10. B.T. Purwanto, International Journal of Pharmacy and Pharmaceutical Sciences, 6(2), 452(2014).

11. K. Dini, Siswandono, B.T. Purwanto, M. Rudyanto, Journal of Chinese Pharmaceutical Sciences, 27(10), 665(2018), DOI:10.5246/jcps.2018.10.07

12. A.J. Jain, and A. Nicholls, Journal Computational Aided Molecular, 22, 133(2008), DOI:10.1007/s. 10822-008-9196-5

13. Dassault Systèmes BIOVIA, 'Discovery Studio Modeling Environment, San Diego: Dassault Systèmes(2018)

14. G. Li, L.A. Hasvold, Z.F. Zao, G.T. Wang, S.L. Gwaltney, J. Patel, P. Kovar, R.B. Credo, Z. Chen, H. Zhang, C. Park, H. Sham, T. Sowin, S.H. Rosenberg, N.H. Lin, Bioorganic and Medicinal Chemistry Letters, 16, 2293(2006), DOI:10.1016/j.bmc/ 2006.01.028

15. M.F. Sanner, Journal of Molecular Graphics and Modelling, 17, 57(1999).

16. S.K. Burley, et al. Nucleic Acids Research 47, D464(2019), DOI:10.1093/nar/gky1004

17. A. Yanuar, Penambatan Molekular: Praktek dan Aplikasi pada Virtual Screening'. Depok: Fakultas Farmasi Universitas Indonesia, p. 1-3(2012)

18. A. Fischer, M. Sellner, S. Neranjan, M.A. Lill, and M. Smieško, ChemRxiv, Preprint (2020), DOI:10.26434/CHEMRXIV. 11923239.V1

19. R. Salomon-Ferrer, D.A. Case and R.C. Walker, Wiley Interdisciplinary Reviews: Computational Molecular Science, 3(2), 198(2013), DOI:10.1002/wcms.1121

20. E.F. Pettersen, T.D. Goddard, C.C. Huang, G.S. Couch, D.M. Greenblatt, E.C. Meng and T.E. Ferrin, Journal of Computational Chemistry, 25(13), 1605(2004), DOI:10.1002/jcc.20084

21. J. Wang, W. Wang, P.A. Kollman, and D.A. Case, Journal of Molecular Graphics and Modelling, 25(2), 247(2006), DOI:10.1016/ j.jmgm.2005.12.005

22. A. Ghaleb, A. Aomdate, M. Bouachrine, T. Laklifi, A. Sbai, Advance Pharmaceutical Bulletin, 9(1), 84(2019), DOI: 10.15171/apb.2019.011

23. S. Saeed, N. Rashid, P.G. Jones, M. Ali, and R. Hussain, European Journal of Medicinal Chemistry, 45, 1323(2010), DOI:10.1016/j.ejmech.2009.12.06

24. J.M. Mc Murry, Organic Chemistry, $9^{\text {th }}$ Ed., Belmont: Broke/Cole, pp 263-289; 779-851(2016).

25. D. Kesuma, Siswandono, B.T. Purwanto, R. Marcelino, World Journal Pharmaceutical Research, 7(7),70(2018), DOI:10.20959/WJPR 20187-11474

26. Siswandono, R. Widyowati, A. Suryadi, T. Widiandani, D. Prismawan, Rasayan Journal of Chemistry, 13(4), 2559(2020), DOI:10.31788/RJC.2020.1345749

27. B. Purwono, B.A. Nurohmah, P.Z. Fathurrohman, And J. Syahri, Rasayan Journal of Chemistry, 14(1), 94(2021), DOI: 10.31788/RJC.2021.1416088

28. R.M. Silverstein, F.X. Webster, and D.J. Kiemle, Spectrophotometric Identification of Organic Compound, 8th Ed, John Wiley and Sons, Inc., New York. (2015).

29. Ruswanto, A.M. Miftah, D.H. Tjahjono, Siswandono, Proccellia Chemistry, 17, 157(2015). DOI:10.1016/j.proche.2015.12.105

30. C.A. Lipinski, F. Lombardo, B.W. Dominy, P.J. Feeney, Advanced Drug Delivery Reviews, 46(1-3), 3(2001), DOI:10.106/s0169-409x(00)00129

31. P. Imming, Medicinal Chemistry: Definitions and Objectives, Drug Activity Phases, Drug Classification Systems, in C.G. Wermuth, D. Aldous, P. Raboisson, D. Rognan, The Practice of Medicinal Chemistry, 4th ed., Amsterdam: Elsevier Ltd,.(2015), DOI:10.1016/s 0169-409 $\mathrm{x}(00) 00129$

32. D.E. Pires, T.L. Blundell, D.B. Asher, Journal of Medicinal Chemistry, 58(9), 4066(2015), DOI:10.1021/acs.j.med. chem.5600104

[RJC-6196/2020] 